\title{
An Energy Aware Protocol for Cyclic Monitoring Wireless Sensor Networks
}

\author{
Ittipong Khemapech \\ School of Science and Technology \\ University of the Thai Chamber of Commerce \\ Dindaeng, Bangkok, Thailand \\ ittipong_khe@utcc.ac.th
}

\author{
Ishbel Duncan and Alan Miller \\ School of Computer Science \\ University of St Andrews \\ North Haugh, St Andrews, Fife, Scotland, UK \\ \{ishbel.duncan, alan.miller\}@st-andrews.ac.uk
}

\begin{abstract}
The Power \& Reliability Aware Protocol (PoRAP) has been developed to provide efficient communication by means of energy conservation without sacrificing reliability. This has been achieved using direct communication, adaptive transmission power adaptation and intelligent scheduling. The key capabilities of PoRAP make it suitable for environmental and habitat monitoring systems. Several experiments were designed and implemented using TinyOS components located at base station and sources. A set of experimentation was set up to determine power adaptation, achieved reliability and energy conservation. From measuring the Received Signal Strength Indicator, the Packet Reception Rate was estimated. Timing relationships were established to predict delays and to determine the required slot size for a schedule based protocol. Statistical analysis of the derived measurements demonstrated significant communication ranges for both indoor and outdoor environments. Further, by adapting the power, the protocol was able to maintain network performance in an optimal region.
\end{abstract}

Keywords-Wireless Sensor Networks; Energy Aware Protocol; Schedule Based Protocol; Direct Communication; Transmission Power Adaptation; Medium Access Control

\section{INTRODUCTION}

Energy conservation is growing in importance. This paper focuses on the issue of energy conservation within the domain of Wireless Sensor Networks (WSNs) which are an important area of research. Data about the physical environment may be collected from hostile or friendly environments. Data is then transmitted to a destination without the need for communication cables. Protocol development for WSNs differs from the traditional approach where issues like survivability, maximising throughput or reliability have been prioritised. Making energy conservation an important design priority is a new approach.

There are four key issues which should be investigated when developing a protocol for WSNs. Firstly, resource constraint is one of the main challenges as the required power or energy for all operational aspects is often provided by small batteries. Secondly, the specific application requirements, such as reliability and data rate, should be considered. Thirdly, many sensors employ Radio Frequency (RF) for communication. It is difficult to use a model to accurately predict signal strength, even when transmission power and distance are known. Finally, nodes in WSNs communicate via a shared medium. Medium access and utilisation is therefore crucial in protocol development.
PoRAP consists of three main components; direct communication, adaptive transmission power and scheduling. It is developed to provide an efficient data communication in single-hop WSNs where the sources communicate directly with their base station. In order to conserve transmission energy, PoRAP adopts the Transmission Power Control (TPC) approach like [4-6]. The adaptation criterion depends upon the relationship between Received Signal Strength Indicator (RSSI) and Packet Reception Rate (PRR). A schedule based scheme is adopted for the sources' transmissions. It is assumed that nodes will be reporting data regularly back to the base station.

The remaining parts of this paper are organised as follows: Section 2 summarises related work. This includes other work that looks at medium access control protocol for WSNs, transmission power control scheme and single-hop application in WSNs. Section 3 describes PoRAP design and implementation. PoRAP has been implemented on TinyOS 2.0.2. In Section 4 an evaluation of PoRAP is described. The evaluation shows both that transmission energy is saved and the PRR is maintained. Further, clock drifts were measured and statistical analyses were conducted to demonstrate the feasibility of further reduction in listening energy.

\section{RELATED WORK}

This section summarizes related work in three areas which focus on medium access control (MAC), transmission power control (TPC) and single-hop application for WSNs.

\section{A. MAC for WSNs}

Two major schemes including contention and schedule based have been adopted and enhanced for WSNs. Throughput is important in the event based application [9-11] whereas periodic based applications such as habitat and environmental monitoring systems are mainly concerned with lifetime of the battery [12-13].

1) Contention based: Radio communication is considered as a shared medium. ALOHA has been regarded as the basis of later protocol developments which include Carrier Sense Multiple Access (CSMA) [14]. In CSMA with Collision Avoidance, data transmission is randomly delayed if the medium is declared busy. Variations in signal and presence of outliers are used in Berkeley-MAC (B-MAC) to detect channel availability [15]. Reliable data reception is provided in B-MAC by long preamble communication. The RTS/CTS (Request-To-Send/Clear-To-Send) handshake is 
adopted by Sensor-MAC (S-MAC) [16] to mitigate the hidden node problem. A SYNC frame containing scheduling details is used; it is exchanged between a source and its neighbours.

An important source of energy wastage in CSMA is idle listening as the nodes are not switched to sleep mode. A check interval is used in B-MAC and the length of preamble must be at least the check interval. Hence, preamble communication is considered an important overhead in B-MAC. In the case of $\mathrm{S}$-MAC, an active interval is required for the configuration. Control frames are an overhead in S-MAC as they require communication energy.

2) Schedule based: Instead of using carrier sensing, another MAC protocol approach, based upon the schedule based schema, can be used. A node is only able to send within its own time slot. It is switched to sleep mode elsewhere. Hence, data collisions and idle listening can be avoided and minimised. The slotted base approach employed in the Cambridge Ring supports collision avoidance [17-18].

A single and time slotted channel is used for data and control transmissions in Traffic Adaptive Medium Access Protocol (TRAMA) [19]. The random access slots are used for controlling whilst the scheduled access ones are used for data transmissions. Each node has to store and maintain the exchanged schedule. A specific MAC for environmental monitoring WSNs, SEA-MAC, is developed in [20]. By assuming that the sensing schedule is known in advance, the base station recognises and maintains the synchronisation. The schedule information is sent by the base station and the nodes disseminate to their neighbours.

Time synchronisation is crucial in a schedule based protocol as the nodes have to agree upon and follow the predefined schedule. A reference node is thus required. Clock drift occurs when different local clocks run at different speeds. It can be accumulated into seconds and the synchronisation deteriorates. In [21], receivers use the packet's arrival time as a reference for comparing their local clocks. The receivers exchange the measurements and the offsets are then computed. In a sender-receiver based scenario, the synchronisation is conducted at the receiver [22]. Each node firstly assigns itself a level for a spanning tree. Its neighbours assign their levels after receiving the broadcast message. A pair-wise synchronisation is performed and the same processes will be repeated to create a network-wide synchronisation.

\section{B. Transmission Power Control}

Communication accounts for a significant amount of energy. The main concept of the Transmission Power Control (TPC) is to adjust transmission power of a sensor with respect to varying link quality.

Each sensor has to know neighbours located in its communication range. There are two main schemes including broadcasting messages and obtaining the topology from the routing protocol. Alternatively, a beacon is broadcast for the discovery [5-6].

Several measurements such as the Received Signal Strength Indicator (RSSI) and the Link Quality Indication (LQI) are used for the feedback mechanism to let the transmitting node know the current link quality and the power required to reach its destination node [5-6]. Other attributes were used as the feedback such as the Packet Reception Rate (PRR) [4] and life messages [23].

Once the minimum transmission power for each pair of sensors has been found, it will be used for future transmissions. Each node has additional costs on storing and maintaining the neighbours table. Further, the path which consumes the least energy will be calculated and then used for an end-to-end data delivery. Link quality metrics changes over time. It should be monitored to reflect the current link quality. Such mechanisms require additional data delivery and computation.

\section{Single-hop Applications in WSNs}

There are currently several applications specifically developed for the single-hop WSNs. The main limitation of the single-hop is communication range. In order to apply the single-hop to large area, several clusters are created and single-hop may be used in each cluster.

An energy balanced protocol for the single-hop is developed in [24]. There are two phases of data routing. Firstly, the packet is routed to suitable cluster which contains the destination node and secondly, the packet is redistributed within the cluster in single-hop. The packets will be routed to achieve an equal distribution of workload in terms of communication.

Single-hop was used in several habitat and environmental monitoring systems [12-13,25]. In such systems, the sensors send their data back to the base station every minutes or hours. In [12-13], a duty cycle of approximately $1 \%$ is required. The system in [12] is divided into three tiers. Single-hop was used in each communication patch which is in the first tier. The sources sent every 5 minutes and the targeted lifetime was 9 months. In [25], the sensors were located within the ice and collected data such as temperature, strain and pressure every 4 hours for 15 seconds.

Several energy-efficient algorithms such as sorting [26] and reprogramming [27] have been developed for single-hop WSNs. A single-hop, and time-synchronised WSN is assumed in [26]. The remaining power of each sensor is sorted to find the maximum. The sensor which owns the highest remaining power will be assigned to report data in the next transmissions. Reliable data delivery is important in reprogramming the sensors [27]. New code or amendment to the existing code may be required during the sensor's lifetime. The single-hop is more suitable when the link is unreliable and linear or approximately linear topology is used.

\section{DESIGN AND IMPLEMENTATION}

This section describes the design and implementation of the Power \& Reliability Aware Protocol (PoRAP). Some of the existing TinyOS 2.0.2 components are used and modified to achieve the protocol's objectives.

\section{A. Key Capabilities of PoRAP}

PoRAP consists of three main capabilities which are described as follows: 
1) Schedule based protocol: In PoRAP, the base station may be connected to several sensors which require access to a shared medium. The MAC approach attempts collision avoidance, one of the main sources of power wastage in the shared medium system.

PoRAP employ the schedule based scheme in which each node is assigned a specific duration to use the shared medium. Sources listen to the base station only once in a frame which represents a communication cycle. Idle listening is therefore minimised. Moreover, data collisions at the base station can be avoided as there is only one source sending at a time.

Centralised scheduling control by the base station is also feasible in PoRAP. The base station broadcasts a packet to all sources located in its range. Slot information such as the number of slots, slot length and start time of the first slot are included in the payload. Once the first frame is finished, the base station broadcasts again with the transmission power adaptation notification.

2) Communication power conservation: The power adaptation mechanisms in PoRAP do not require historic entries of RSSI and associated TX. The main reason is the limitation of buffering capacity of the radio chip. The base station should support a significant number of sources. In the CC2420 radio, the maximum buffer size is 128 bytes. Some bytes are required for the header and other controlling details. Only two bits are used to notify the power adaptation. The RSSI-PRR relationship provided in [6,29] can be used for adaptation as it suggests the operating region for WSNs. An increase in transmission power does not increase PRR and a decrease in the power does not compromise PRR in the operating region. In the case of power adaptation, the base station sets particular bits to notify the source. The sources get the notification bits and set their transmission power.

3) Link quality monitoring: Radio communication uses air as the transmission medium. There are several attributes ranging from differences in hardware components to environmental factors such as physical barriers which affect signal attenuation. Received signal strength estimation is difficult as sensors can be placed in various areas of interest. An estimation model should not only determine the distance between sender and receiver as an input, but location should also be concerned.

Two link quality metrics are used in PoRAP. The RSSI is obtained by the radio chip whilst the PRR is specified by the applications. The relationship between RSSI and PRR can relate the application requirement to the observed link quality. As shown in [6,29], a clear relationship between the two metrics is established. The PRR steeply increases with the RSSI up to a certain point. The PRR is then stable over a range of RSSI so the lower RSSI or TX can be used to obtain the required PRR. However, it was found that RSSI, LQI and PRR change over time and the metrics should be continually monitored [30].

The range of required RSSI is obtained from the reliability requirement and the RSSI-PRR relationship. This range is noted by the base station. Upon data reception, the base station measures the RSSI and compares it to the RSSI thresholds. The adaptation bits are set with respect to the comparison result. There are three available patterns of bit settings; the transmission power will be increased if the measured RSSI is lower than require and it will be decreased if the RSSI is higher. The sources will be notified to retain the current power if the RSSI is within the range.

\section{B. PoRAP Architecture}

Input to PoRAP comes from two external components, the user/application and the monitored phenomenon. PoRAP notes the duty cycle and data loss requirements. The sensed data is another input and it will be sent from the source to the base station. In order to achieve the set goals, the base station controls the sources whereas the sources send data to the base station. The required functionalities of the base station and the sources must be stated. The interactions between them are described and they are used to address the required components within the source and the base station. Moreover, the interactions between such components are also given in this section.

1) Overview of PoRAP: Four main components are addressed in PoRAP; the user/application, the sensed phenomenon, the base station and the sources. As WSNs are application specific, the user/application has its own set of requirements. The base station directly interacts with the user/application whilst the sources collect physical data directly from the phenomenon. The functionalities required at the base station and source can be listed as follows:

\section{Base station:}

There are two main two main functionalities of the base station. Firstly, it notes the requirements of the user/application. PoRAP aims at the low duty cycle application where the key objective is power conservation instead of throughput. Examples of this application category are habitat and environmental monitoring systems. Secondly, it controls the source's operation. The base station determines whether transmission power used by the source needs to be adjusted by looking at the measured RSSI. The communication cycle of each source is scheduled in order to avoid data collision and minimise idle listening.

\section{Source:}

The source is responsible for two operations. Firstly, it collects the physical data. The processes of data collection are outside the scope of this study. Secondly, it transmits data to the base station. After receiving the control information, the source sets two parameters; it synchronises the communication schedule to know when to start the radio for control reception and data transmission and further, the source adapts its transmission power level according to the included notification. If a lower power can be used, a significant amount of transmission power can be conserved. 


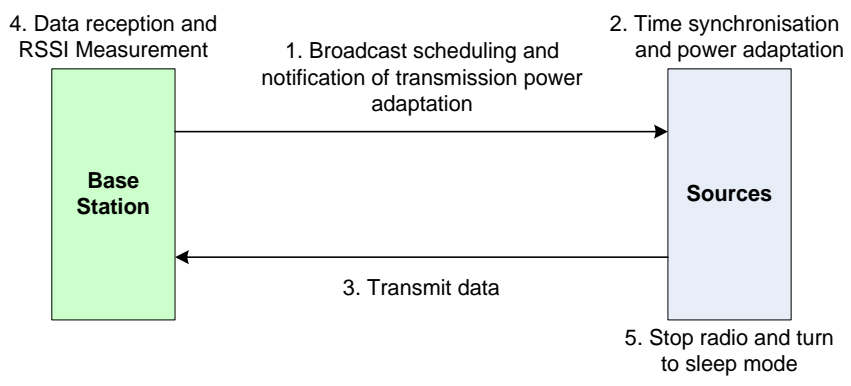

Fig. 1. Interactions between source and base station

Several interactions between the source and base station are required to achieve the functional requirements and they are addressed in Fig. 1 as follows:

1. In PoRAP, the control packet is broadcast by the base station and includes scheduling and power adaptation notification and is broadcast to the sources using the maximum power level.

2. Once the control packet is received by the source, the information on scheduling and notification is read. The source synchronises its schedule with the other nodes and adjusts its transmission power.

3. The source then waits for its slot to conduct data transmission using the adjusted transmission power. The radio must be started for communication.

4. The base station measures the RSSI during data reception. The observed RSSI is compared to the desired range which includes the minimum and maximum values. The selected RSSI should be obtained from the region where significant stability in the PRR is observed. The base station then decides whether transmission power adaptation is required. The notification is set accordingly.

5. The source stops its radio after transmission to save power. The amount of power consumption is the least when the source is in sleep mode. Timing is required for the source to start the radio again for the next communication cycle.

2) Components: PoRAP takes components from TinyOS and adds some further modifications. The main components are determined from the interactions including the user/application, the observed phenomenon, the base station and the source.

Radio: sensor employs the radio communication for wirelessly communicating with its neighbours or destinations. The radio has four major functions. Firstly, it makes data communications between the nodes occur. Secondly, the data is buffered in the radio prior to transmission and after reception. The buffering capacity is limited and dependent upon the radio chip [7-8]. The capacity is important to the design of packet structures. Thirdly, the latest radio chip provides the measurement of received signal strength such as Received Signal Strength Indicator (RSSI) and Link Quality Indication (LQI). Finally, programmable transmission power is also supported by the latest radio.

Timer: The transmissions of sources are scheduled. A slot is allocated for each source so that it can send only when its slot arrives. Otherwise, the radio is stopped and the source is switched to sleep mode for minimum energy consumption. A timer is thus required for scheduling the radio start and stop.

Control: This is used to control the other components especially when there is no control mechanism provided. For example, an additional control interface is required for the radio and the interface is used to start and stop the radio.

Memory: Several variables along with their values and measurements are stored in the memory. For example, the required RSSI range obtained from the RSSI-PRR relationship is stored in the memory and will be compared to the observed RSSI to determine whether any transmission power adaptation is required.

Sensor board: This component is crucial for the sensors as it is responsible for collecting the physical data from the environment. The sensor board consists of several sensors such as temperature and humidity

3) Interactions between components: The interactions between the components are described in Fig. 2. The interactions within the base station and source can be separately described as follows:

\section{Base station:}

The requirements are stored in the memory and are used to set the required RSSI range and the data sending rate. The timer is used for scheduling the communications so it also uses this requirement from the application. The required RSSI range can be obtained from the RSSI-PRR relationship. The base station uses the observed RSSI to determine whether power adaptation is required.

The base station's radio is not started or stopped as it has to continually receive the data packets from its sources. Data packet receptions occur after broadcasting the control packet. In PoRAP, power conservation is mainly located at the sources.

\section{Source:}

Prior to transmission, the source determines whether it has to adapt its current power by looking at the notification bits. Thus, the source has to store the current power in the memory. Moreover, the source should recognise the limitations of the transmission power adaptation. The base station may need its source to increase the power even if the maximum has already been reached.

Apart from the power adaptation signaling, the scheduling is also included in the control packet. The local clock of each node may run at different speeds. In PoRAP, the sources synchronise with their base station. The scheduling is also recognised by the timer and control components. Several timers are required as they are responsible for timing the sending and receiving communications.

\section{PoRAP Implementation}

This section describes the implementation of PoRAP. Standard notations provided by the TinyOS community are used [1]. Further, control and data packet structures are given. 


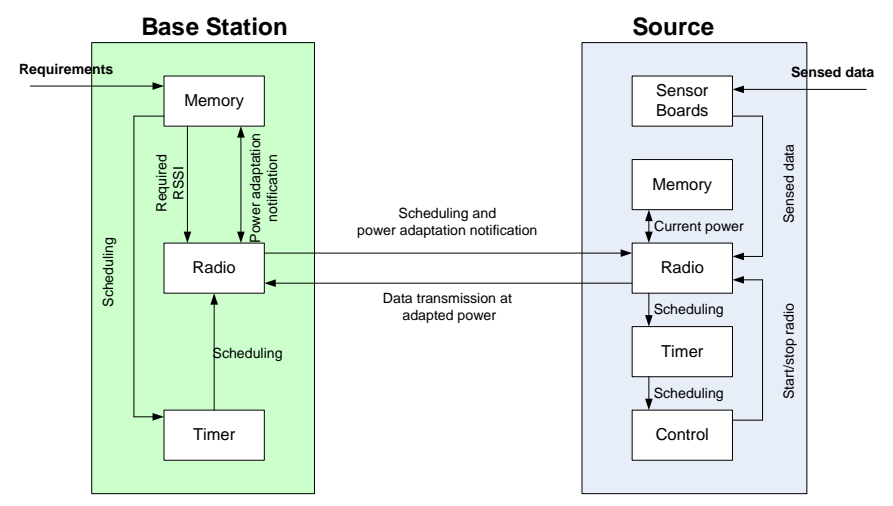

Fig. 2. Interactions between components

1) TinyOS component: TinyOS is written by the nesC programming language which is a $\mathrm{C}$ dialect. A nesC component provides and uses an interface. Provided interfaces represent the functionalities of the component and they can be used by the other components. TinyOS also provides a programming environment so that new protocols or applications can be developed by adopting or modifying several existing components.

\section{Structure of source's component:}

The source module is represented by Sourcec. It uses several interfaces provided from eight components as follows:

Mainc: This component is general and is required in all modules. It provides the Boot interface which is used for booting the module for operation.

ActiveMessage $\boldsymbol{C}$ : This standard message structure is general so that the sensors can understand each other. This component provides a key interface called "SplitControl" which is useful for starting and stopping the hardware. An instance can be used to control specific hardware such as the radio.

cC2420Transmitc: Two important interfaces are provided by this interface. The CC2420Transmit enables the modification to the payload especially when the Start of Frame Delimiter (SFD) of the packet is transmitted. The other interface is RadioTimestamping which is used for obtaining the specific time of data transmission and reception at the MAC layer.

CC2420ActiveMessagec: This component is important to PoRAP as it provides the CC2420Packet interface which enables the transmission power setting.

AMSenderC: This component is generic and an instance called "AMSenderDataC" is created to facilitate data transmission. Two important interfaces are used. Firstly, the Packet interface is used for obtaining the payload part from the whole packet. This is useful for modifying the content in the payload. Secondly, the AMSend is required for message sending by calling the AMSend. send ( ).
AMReceiverc: Like AMSenderC, this component is generic. Its instance, AMReceiverCtrlC, is created to facilitate data reception in PoRAP. The Receive interface is used and several processes are performed when the Receive.receive( ) is signaled.

Alarm32khz32C: is used for timing the waiting and sleeping intervals. Its commands take and return an unsigned 32-bit integer. Two alarm components are thus required. The returned values from the Alarm component must be used for calculating waiting and sleeping intervals. The 32-bit Alarm must be converted to Timer for such calculations.

AlarmToTimerc: Two instances of this component, WaitTimer and SleepTimer, are created for the source. They are respectively used for timing the waiting and sleeping intervals. The MotewaitTimer is an interface of the WaitTimer and the MotesleepTimer is an interface of SleepTimer. The WaitTimer interface is started by calling the MotewaitTimer.fired() command. It is called after the source has received the control packet whereas the SleepTimer is called after the data packet has been sent.

\section{Structure of base station's component:}

The base station module, BaseP, can be considered as an enhancement of the BaseStation application in the TinyOS 2.0.2. The BaseStation is a basic TinyOS utility application. It acts as a bridge between the serial port and the radio network. It is developed to forward the data received by the radio to the serial port and then show it on the computer screen. Additional work has been conducted to manage the buffer of the radio unit. Several enhancements are made to the BaseStation as follows:

- The base station can broadcast its control packet to the fixed set of sources which are located within its communication range.

- The base station is able to measure the Received Signal Strength Indicator (RSSI) during data reception.

- The base station compares the observed RSSI to the bounds and sets the notification bits in order to signal the transmission power adaptation to the source.

- The base station is able to schedule the control packet broadcast to maintain time synchronisation between itself and the sources.

In order to achieve the enhancements stated previously, additional components are required apart from the notification bit settings as follows:

AMSenderC: An instance of this component called "AMSenderCtrlC" is created to facilitate control packet transmission. Two important interfaces are used by BaseP. Firstly, the Packet interface is used for obtaining the payload part from the control packet for message modifications. Secondly, the AMSend is required for message sending by calling the AMSend. send ( ). 
cc2420ActiveMessageC: This component is important to PoRAP as it provides the CC2420Packet interface which enables RSSI measurement. This can be conducted by calling CC2420Packet.getRssi(msg). Further, the command called getRssi ( )accepts an argument which is the message being received.

Alarm32khz32C: is used for timing the control packet broadcast. An alarm component is thus required. The explanations given in the previous part for the source apply to the base station.

AlarmToTimerc: An instance of this component, BcastTimer, is created. The fired( ) command is called after the control packet is sent.

The main TinyOS components used in PoRAP are those which provide transmission power setting, RSSI measurements, scheduling, modification to payload, sending and transmitting. The base station module is an enhancement of the existing BaseStation utility application in TinyOS 2.0.2. The main enhancements include data transmission, RSSI measurements and scheduling.

2) Control and data packet structures: Two packet formats are required in PoRAP. The control packet is used in the control and setup phase. It contains essential information for transmission power adaptation and time synchronisation. The data packet is used to deliver the sensed phenomenon to the base station. Details of both packet structures are given in this section.

\section{Control packet:}

The control packet consists of 5 fields. The first byte, base_id, represents the base station's address. The address can be assigned at installation time. The no_alloc_slot demonstrates the number of allocated slots and is stored in an 8-bit field. Slot length is contained in the 16-bit slot_length. The time when the first data slot is started is demonstrated in the 16-bit slot_start.

TABLE I

THREE POSSIBLE POWER ADAPTATION PATTERNS

\begin{tabular}{|c|l|}
\hline Pattern & \multicolumn{1}{c|}{ Meaning } \\
\hline 00 & RSSI is in the range, keep TX the same \\
\hline 01 & RSSI is higher than required, decrease TX \\
\hline 10 & RSSI is lower than required, increase TX \\
\hline
\end{tabular}

Finally, the tx_adapt [s] is used to notify the sources whether transmission power adaptation is required. The default payload size in the message_t abstract data structure is set to 28 bytes. However, the maximum payload for the CC2420 is approximately 117 bytes. The former fields take 6 bytes. The remaining size for tx_adapt [s] depends upon the number of sources. Two bits are arranged for a source and therefore one byte supports four sources. Three possible patterns of two bits for the notification of transmission power adaptation are summarised in Table 1.

\section{Data packet:}

The data packet consists of 2 fields. The 8-bit source_id field represents the sensor address which is defined at the installation time. Finally, the data is stored in data [q] where $q$ is the number of bytes used for storing data. The width of data field is defined by the WSN application.

\section{IV.EVALUATION}

This section describes PoRAP evaluation and the experiments set up to evaluate the energy conservation of the protocol. PoRAP was implemented on Tmote Sky [2] which employs CC2420 radio unit [7]. The work addresses four subjects. Firstly, the distance over which direct communication is possible is analysed. Secondly, a comparison of the energy consumption for direct and multihop communication is made. The effects of distance between nodes and densities are studied. Thirdly, an evaluation of the feasibility and benefit of adaptive power transmission is performed to discover the optimal region where transmission energy is conserved and a reliability of nearly $100 \%$ is maintained. Finally, an evaluation of clock drift is performed.

\section{A. Estimation of Communication Ranges}

Using results based on free space model, our experimental studies and in [3], models for predicting the communication ranges by non-linear regression analysis were developed for indoor and outdoor environments. In our measurements, in total 10 different distances ranging from 1 to $20 \mathrm{~m}$ were used to measure the RSSI. The experiment was repeated 50 times for each transmission power setting. The average RSSI was computed and plotted against the distance. Similar procedures were conducted for the free space model and the results in [3]. For all cases, the logarithmic approach provided the highest R-square value which describes how well a regression line estimates the set of real data.

The RSSI of $-95 \mathrm{dBm}$ is used to consider the feasible communication range as the CC2420 does not report the observed RSSI below -95dBm. Different transceiver such as CC1000 [8] provides different minimum reported RSSI value. Therefore, the feasible ranges are obtained from the interceptions between the $-95 \mathrm{dBm}$ horizontal line and the curve between distance and reception strength. However, packet losses are likely to occur if a lower transmission power is used to produce the RSSI of $-95 \mathrm{dBm}$. One of the key requirements in data delivery in the network is to minimise data losses. According to the RSSI-PRR relationships established in [6,29-30], a RSSI value of $-85 \mathrm{dBm}$ or higher often produces the PRR of nearly $100 \%$. Thus, both RSSI values of -95 and $-85 \mathrm{dBm}$ are used for the estimations. The results are shown in Table 2. 
TABLE II

FEASIBLE AND MEASURED COMMUNICATION RANGES

\begin{tabular}{|c|c|c|c|c|c|c|c|c|}
\hline \multicolumn{2}{|c|}{ Transmission Power } & \multicolumn{6}{|c|}{ Estimated Communication Ranges (m) } & \multirow{3}{*}{$\begin{array}{c}\text { Maximum Distances (m) from } \\
\text { Measurements }\end{array}$} \\
\hline \multirow{2}{*}{$(\mathrm{dBm})$} & \multirow{2}{*}{ Level } & \multicolumn{2}{|c|}{ Free Space Model } & \multicolumn{2}{|c|}{ Indoor } & \multicolumn{2}{|c|}{ Outdoor } & \\
\hline & & $-95 \mathrm{dBm}$ & $-85 \mathrm{dBm}$ & $-95 \mathrm{dBm}$ & $-85 \mathrm{dBm}$ & $-95 \mathrm{dBm}$ & $-85 \mathrm{dBm}$ & \\
\hline-25 & 3 & 65 & 20 & 10 & 2.5 & 15 & 10 & $1.5-4.0$ \\
\hline-15 & 7 & 200 & 70 & 20 & 8 & 70 & 29 & $5.5-7.0$ \\
\hline-10 & 11 & 390 & 120 & 32 & 12.5 & 130 & 48 & $6.5-10.5$ \\
\hline-7 & 15 & 500 & 180 & 45 & 18 & 190 & 67 & $8.5+$ \\
\hline-5 & 19 & 650 & 230 & 65 & 24 & 245 & 85 & $9.0+$ \\
\hline-3 & 23 & 870 & 300 & 74 & 29 & 305 & 105 & $9.5^{+}$ \\
\hline-1 & 27 & 1,000 & 380 & 80 & 33 & 360 & 124 & $9.5^{+}$ \\
\hline 0 & 31 & $1,000+$ & 420 & 96 & 38 & 420 & 143 & $9.5^{+}$ \\
\hline
\end{tabular}

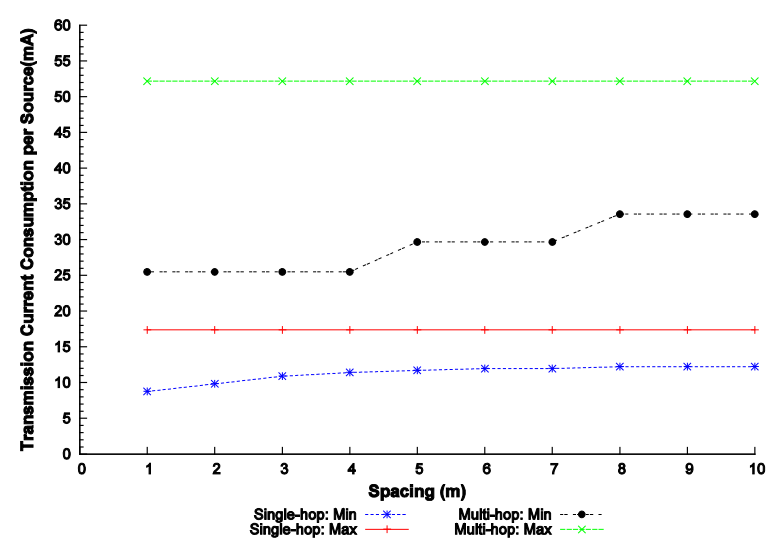

Fig. 3. Effects of spacing on transmission current consumption

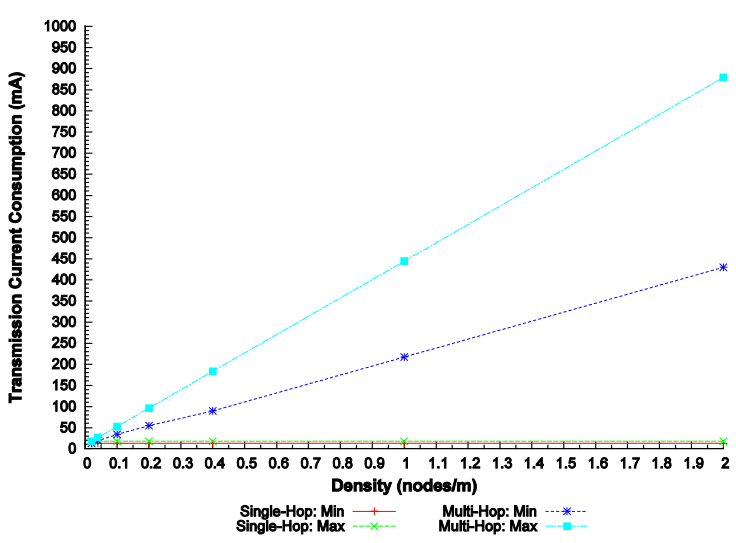

Fig. 4. Effects of node density on transmission current consumption

TABLE III

CONSERVED TRANSMitTing CURRENT AND DATA PACKET LOSS

\begin{tabular}{|c|c|c|c|c|c|c|c|c|c|c|}
\hline \multirow{2}{*}{$\begin{array}{c}\text { Dist. } \\
(\mathrm{m})\end{array}$} & \multicolumn{2}{|c|}{$-90<\mathrm{RSSI}<-80$} & \multicolumn{2}{|c|}{$-80<\mathrm{RSSI}<-70$} & \multicolumn{2}{|c|}{$-70<\mathrm{RSSI}<-60$} & \multicolumn{2}{|c|}{$-60<\mathrm{RSSI}<-50$} & \multicolumn{2}{c|}{ Max TX } \\
\cline { 2 - 13 } & $\begin{array}{c}\text { Saved } \\
\text { Trans } \\
\text { Current }\end{array}$ & $\begin{array}{c}\text { Packet } \\
\text { Loss } \\
(\%)\end{array}$ & $\begin{array}{c}\text { Saved } \\
\text { Trans } \\
\text { Current }\end{array}$ & $\begin{array}{c}\text { Packet } \\
\text { Loss } \\
(\%)\end{array}$ & $\begin{array}{c}\text { Saved } \\
\text { Trans } \\
\text { Current }\end{array}$ & $\begin{array}{c}\text { Packet } \\
\text { Loss } \\
(\%)\end{array}$ & $\begin{array}{c}\text { Saved } \\
\text { Trans } \\
\text { Current }\end{array}$ & $\begin{array}{c}\text { Packet } \\
\text { Loss } \\
(\%)\end{array}$ & $\begin{array}{c}\text { Saved } \\
\text { Trans } \\
\text { Current }\end{array}$ & $\begin{array}{c}\text { Packet } \\
\text { Loss } \\
(\%)\end{array}$ \\
\hline 1 & 51.2 & 0 & 51.2 & 0 & 51.2 & 0 & 35.6 & 0 & 0 & 0 \\
\hline 2 & 51.2 & 0.3 & 35.6 & 0.7 & 0 & 0 & 0 & 0 & 0 & 0 \\
\hline 4 & 43.1 & 2.3 & 43.1 & 0.7 & 28.2 & 0 & 0 & 0 & 0 & 0 \\
\hline 6 & 43.1 & 4.7 & 28.2 & 0 & 0 & 0.3 & 0 & 0 & 0 & 0 \\
\hline 8 & 51.2 & 5 & 0 & 0.7 & 0 & 0.3 & 0 & 0 & 0 & 0 \\
\hline 10 & 51.2 & 5.3 & 35.6 & 0 & 0 & 0 & 0 & 0 & 0 & 0 \\
\hline 12 & 51.2 & 5.7 & 20.1 & 0 & 0 & 0 & 0 & 0 & 0 & 0 \\
\hline 14 & 0 & 14 & 28.2 & 0 & 0 & 0 & 0 & 0.4 & 0 & 3.7 \\
\hline 16 & 28.2 & 5.7 & 20.1 & 0 & 0 & 0 & 0 & 0 & 0 & 1.2 \\
\hline 20 & 43.1 & 3.7 & 0 & 0.7 & 0 & 0 & 0 & 1.2 & 0 & 2.1 \\
\hline
\end{tabular}

TABLE IV

VARIATIONS IN CLOCK DRIFT

\begin{tabular}{|c|c|c|c|c|c|c|}
\hline \multirow{2}{*}{ Duration } & \multirow{2}{*}{ Ticks $\left(* 10^{6}\right)$} & \multicolumn{2}{|c|}{ Range of Variations } & Width (ticks) & 20 ppm & Saved (\%) \\
\hline 5 minutes & 9.8 & -27 & 36 & 63 & 68 & 396 \\
\hline 10 minutes & 19.6 & -47 & 37 & 84 & 2,360 & 79 \\
\hline 1 hour & 118 & -249 & 200 & 449 & 56,620 & 95 \\
\hline 1 day & 2,831 & -1620 & 940 & 2560 & & 95 \\
\hline
\end{tabular}


According to Table 2, shorter communication ranges are achieved if $-85 \mathrm{dBm}$ is required instead of $-95 \mathrm{dBm}$. This is because the reception strength decreases with longer distances. The free space model gives significant ranges as no barriers and good weather are assumed in the model. The estimated values based upon $-85 \mathrm{dBm}$ indicate that direct communication can be applied to wireless sensor networks as the sensor has up to $38 \mathrm{~m}$ indoor and $143 \mathrm{~m}$ outdoor ranges whilst the packet losses are minimised.

\section{B. Comparison of Single and Multi-hop}

Two studies were conducted where the energy requirements for single and multi hop communications were compared. In the first analysis the distance between nodes varied between one and ten metres. The transmission power required for distances was obtained through measurement and is shown in Table 2. The base station remained at the same location throughout the experiment whilst three sensors were placed at ten different locations, 1, 2, 3, 4, 5, 7, 10, 13, 16 and 20m. Each power adaptation cycle was ended after the maximum power had been reached. The sensors transmitted a packet every second. At each power setting, 50 packets were sent. In the second analysis the effect of node density was examined.

1) Effects of distances between sources: A 5-hop topology is used to investigate how the distance between sources or spacing affects the current consumption required for single and multi-hop communications. Each source sends a packet which has to be received by all intermediate nodes in the multi-hop case. However, the packet is transmitted directly to the base station in the single-hop case. The spacing between nodes is set between 1 and $10 \mathrm{~m}$. The current required for each distance is obtained from the measurements.

The minimum current shown in Fig. 3 is based upon the experimental results. There may be some circumstances in which a specific transmission power obtained from Table 2 cannot produce the expected RSSI. A higher power is then required for transmission. The maximum power of $17.4 \mathrm{~mA}$ is therefore included in this study. For the single-hop, the maximum current consumption used for transmission per source is $(17.4 \mathrm{~mA}$ per transmission * 5 transmissions / 5 sources) or $17.4 \mathrm{~mA}$. However, the necessary power is (17.4mA per transmission * 15 transmissions / 5 sources) or $52.2 \mathrm{~mA}$ in the case of multi-hop communication.

According to Fig. 3, the amount of current consumption per source increases with the distance between nodes as a higher power is required. The current required approaches the maximum level at greater source spacing. The singlehop requires nearly one-third of the multi-hop current. The main reason is that higher transmissions are required for multi-hop communication. Note that the above results are obtained from the transmission only. An additional 10 receptions are required by the sources in the multi-hop.

2) Effects of source densities: A network consisting of seven different sources uniformly scattered over a line topology which spans a distance of $50 \mathrm{~m}$ is used. Each source sends a packet which has to be received by all intermediate nodes in the case of multi-hop. The numbers of sources, $n$, are set to $1,2,5,10,20,50$ and 100 . The corresponding densities are defined as the number of sources per metre; therefore the densities are $0.02,0.04,0.1$, $0.2,0.4,1$ and 2 . Both the minimum current based upon the experimental results and maximum current based upon the full transmission power capability are calculated and shown in Fig. 4.

The results demonstrate a significant benefit of direct communication over the multi-hop scenario. The benefit is higher for a denser network in comparison to the required message forwarding of a multi-hop network. However, a source may not be able to conduct direct communication at $50 \mathrm{~m}$. According to Table 2, a power setting of $-7 \mathrm{dBm}$ or lower can be used for a $10 \mathrm{~m}$ range which means that 5 sources are required in the topology. Each source consumes approximately $12.24 \mathrm{~mA}$ compared to the $33.6 \mathrm{~mA}$ used by the multi-hop. Hence, almost two-thirds of the transmitting current can be conserved.

\section{Transmission Power Adaptation, Reliability and Energy Conservation}

The previous section demonstrated the viability of direct communication in WSNs. This section describes experimental studies which test the power adaptation capability of PoRAP. In total 20 Tmote Sky sources were placed at 20 separate locations with 14 different distances to address the effects of location. A Tmote Sky base station broadcasts the control packet at the maximum power at the start of each communication cycle. Each source is allocated to a time slot when it can send. After the control packet has been received, a source transmits its data at the maximum power. The base station receives data and decides whether the current power of each source requires adaptation. The communication proceeds for 1,000 cycles. The source should correctly adjust its power corresponding to the notification generated by the base station. The results shown in [30] demonstrate that PoRAP adjusts the transmission power correctly. Most of RSSI measurements are within the required range.

Another experiment was carried out to determine the relationship between RSSI settings, PRR and energy conservation. In total 10 distances including $1,2,4,6,8,10$, $12,14,16$ and $20 \mathrm{~m}$ were used. The minimum RSSI thresholds were set to $-90,-80,-70$ and $-60 \mathrm{dBm}$ whereas the corresponding maximum thresholds were $-80,-70,-60$ and $-50 \mathrm{dBm}$, respectively. The power is not adapted if the measured RSSI is between the thresholds and the aim is to obtain nearly 100\% PRR. Each sensor transmitted every 5 minutes and the experiment lasted for 24 hours.

Table 3 shows the percentage of conserved transmitting current and data packet loss at various distances and RSSI settings. The medians of transmission power used by the motes are used for the calculations. The required current for transmission power is obtained from [7]. The "Max TX" column represents that the motes always transmitted at the maximum power. It is used as a reference to calculate the amount of conserved power. 
According to Table 3, lower RSSI settings result in a higher percentage of packet loss and conserved transmitting power. Lower power is used to produce the required RSSI range. A significant amount of power up to $50 \%$ can be conserved. However, the highest packet loss is obtained when the RSSI is between -90 and $-80 \mathrm{dBm}$. One of the key requirements in the development of a network protocol is to minimise the data loss. This can be gained by always transmitting at the maximum power. The "Max TX" column illustrates this scenario. No power is saved and the packet losses are low. However, it is feasible to use lower power without unnecessary data losses.

For example, the RSSI between -60 and $-50 \mathrm{dBm}$ produced a $35.6 \%$ of conserved power at a $1 \mathrm{~m}$ distance with low data losses at greater distances. Higher power conservation is achieved if a lower RSSI is set. The appropriate RSSI settings mainly depend upon the reliability requirement. Assuming that a network topology consisting of 10 sources located at 10 different distances as shown in Table 3, the application requires at least $99 \%$ of reliability at the base station. The maximum power can be used at all sources but no power is saved. The appropriate RSSI setting is between -80 and $-70 \mathrm{dBm}$. An average power saving of $26.2 \%$ per source is achieved. That is, with such a RSSI setting, the sources use an average $73.8 \%$ of the maximum power.

\section{Measurement of Clock Drift}

PoRAP adopts the schedule based approach where the communication frame is divided into several time slots. Data collisions and idle listening can thus be avoided and minimised. A sensor has an oscillator which generates timing signals or ticks. The number of ticks generated in 1 second depends upon the timer interface provided in TinyOS. In this study, a 32-KHz clock is selected and provides 32,768 ticks per second. Clock drift occurs as a result of uncertainty in the ticking rate because different local clocks may run at different speeds. Clock drift may accumulate and time synchronisation is no longer maintained. Clock drift is crucial in a schedule-based system like PoRAP. A control packet is therefore broadcast at the beginning of each frame or communication cycle to maintain synchronisation.

Clock drifts were also measured in [30]. For each control packet delivery, the times when its Start of Frame Delimiter (SFD) is transmitted at the base station and received by the sources are measured and stored in the control and data packets. The difference between two local times is computed by the base station after the data packet is received. Additional bytes are required for these timestamps. The clock drift is defined as the difference between the two successive differences in the local clocks. In the case where clock drift does not occur, the time differences should be the same.

The measurements shown in Fig. 5 demonstrate that clock drift can be accounted for in the scheduling algorithm as it happens in a predictable way by looking at its median and variation. Hence, by measuring variation in clock drift the accuracy with which scheduling can occur is established. The base station monitors the relative clock drift to each of the sources. Such measurements are broadcasted to the sources and they can adjust their scheduling locally to keep the idle listening period minimum.

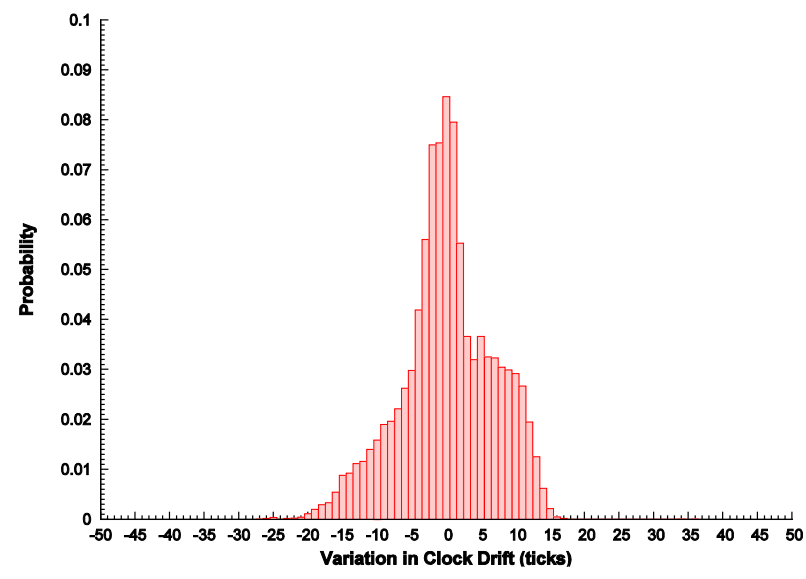

Fig. 5. Distribution in variations in clock drifts

The same computational procedures were repeated to investigate how variations are affected by the durations between two consecutive transmissions. Apart from being hardware dependent and non-deterministic, clock drift also depends upon duration between transmissions. The " 20 ppm" column in Table 4 demonstrates the reserved duration which is recommended by the datasheet [31]. By knowing the drift medians, the motes can adjust their schedules in order to synchronise with the base station. The motes start their radios later if their clocks are running more quickly and they can be in sleep mode longer. The idle listening period is therefore reduced. Therefore up to $95 \%$ of idle listening, due to reserved time for clock drift, can be reduced. The sources spend less energy on idle listening whilst time synchronisation is maintained.

\section{CONCLUSION AND Future WORK}

The development of the Power \& Reliability Aware Protocol (PoRAP) is presented in this paper and its main objective is to provide an efficient data communication by means of energy conservation whilst reliability is maintained. Its three key elements include direct communication, adaptive transmission power and intelligent scheduling. The estimated indoor and outdoor ranges demonstrate the feasibility of direct communication at moderate distances. With adaptive transmission power and intelligent scheduling, the power consumption is minimised as a result of a lower transmitting power, collision avoidance and minimised idle listening without unnecessary data losses.

The key capabilities of PoRAP make it suitable for use in the periodic-based WSN applications with regular reporting patterns where maximising bandwidth is not the 
prime concern. PoRAP thus applies to some of the applications such as environmental and habitat monitoring where the sources often remain at their positions throughout the operation. Slots are allocated to the sources for data transmissions. In PoRAP, it is assumed that the number of allocated slots is equal to that of sources. A low duty cycle application is more efficient using PoRAP when the percentage of slot usage is high. However, PoRAP is not applicable if a source has to wait longer until the next cycle is started. Therefore, a limitation of PoRAP arises when there is a high slot overhead because there are many sources in the network.

As direct communication is limited by the sensor's communication range, single-hop WSNs cannot be used in a large area. A concept of multiple base station system can be applied. Multiple base stations are scattered over the target area. The processes required for this type of system are listed and discussed. The master base station is defined as the final destination for data and it also controls the other base stations by broadcasting master control packets which include scheduling information. The source-to-base station traffic can take place simultaneously by using multi-channel communications. Hence, traffic interference can be reduced.

\section{REFERENCES}

[1] TinyOS. DOI= http:www.tinyos.net

[2] Tmote Sky Datasheet. DOI=http://www.moteiv.com/products/docs/ tmote-sky-datasheet.pdf

[3] Stoyanova, T., Kerasiotis, F., Prayati, A. and Papadopoulos, G. 2007. Evaluation of impact factors on RSS accuracy for localization and tracking applications. In Proceedings of the MobiWac'07, Chania, Crete Island, Greece, October 2007, pp. 9-16.

[4] Son, D., Krishnamachari, B. and Heidemann, J. 2004. Experimental study of the effects of transmission power control and blacklisting in wireless sensor networks. In Proceedings of the IEEE SECON, October 2004

[5] Jeong, J., Cullar, D. E. and Oh, J. H. 2005. Empirical analysis of transmission power control algorithms for wireless sensor networks. University of California Berkeley. Technical Report No. EECS2005-16, November 2005

[6] Lin, S., Zhang, J., Zhou, G., Gu, L., He, T. and Stankovic, J. A. 2006. ATPC: Adaptive transmission power control for wireless sensor networks. In Proceedings of ACM SenSys'06, Boulder, Colorado, USA, November 2006

[7] Datasheet - 2.4 GHz IEEE 802.15.4 / Zigbee-ready RF transceiver. DOI= http://focus.ti.com//lit/ds/symlink/cc2420.pdf

[8] Datasheet - CC1000 single chip very low power RF transceiver. DOI= http://focus.ti.com//lit/ds/symlink/cc1000.pdf

[9] Arora, A., Dutta, P., Bupat, S., Kulathumani, V., Zhang, H., Naik, V., Mittal, V., Cao, M., Demirbas, M., Gouda, M., Choi, Y., Herman, T., Kulkarni, S., Arumugam, U., Nesterenko, M., Vora, A. and Miyashita, M. 2004. A line in the sand: A wireless sensor network for target detection, classification, and tracking. Computer Networks: The International Journal of Computer and Telecommunication Networking, 2004, pp. 605-634

[10] Simon, G., Balogh, G., Pap, G., Maróti, M., Kusy, B., Sallai, J., Lédeczi, Á., Nádas, A. and Frampton, K. 2004. Sensor networkbased countersniper system. In Proceedings of ACM SenSys'04, Maryland, USA, November 2004

[11] Allen, G. W., Lorincz, K., Ruiz, M., Marcillo, O., Johnson, J., Lees, J. and Welsh, M. 2006. Deploying a wireless sensor network on an active volcano. IEEE Internet Computing. 12, March/April 2006, pp. 18-25

[12] Mainwaring, A., Polastre, J., Szewczyk, R., Culler, D. and Anderson, J. 2002. Wireless sensor networks for habitat monitoring. WSNA'02, Georgia, USA, 2002

[13] Tolle, G., Polastre, J., Szewczyk, R., Culler, D., Turner, N., Tu, K., Burgess, S., Dawson, T., Buonadonna, P., Gay, D. and Hong, W. 2005. A macroscope in the redwoods. In Proceedings of ACM SenSys'05, November 2005

[14] Abramson, N. 1970. The ALOHA System - Another alternative for computer communications. In Proceedings of the Fall Joint Computer Conference, November, 1970

[15] Polastre, J., Hill, J. and Culler, D. 2004. Versatile low power media access for wireless sensor networks. In Proceedings of ACM SenSys'04, Maryland, USA, November 2004

[16] Ye, W., Heidemann, J. and Estrin, D. 2003. Medium access control with coordinated, adaptive sleeping for wireless sensor networks.USC/ISI Technical Report ISI-TR-567, 2003

[17] Needham, R. M. 1979. System aspects of the Cambridge Ring. ACM Symposium on Operating Systems Principles, 1979

[18] King, P. J. B. and Mitrani, I. 1982. Modelling the Cambridge Ring. In Proceedings of the ACM SIGMETRICS Conference on Measurement and Modeling of Computer Systems, 1982

[19] Rajendran, V., Obraczka, K. and Garcia-Luna-Aceves, J. J. 2003. Energy-efficient, collision-free medium access control for wireless sensor networks. In Proceedings of ACM SenSys'03, November 2003

[20] Erazo, M. A. and Qian, Y. 2007. SEA-MAC: A simple energy aware MAC protocol for wireless sensor networks for environmental monitoring applications. In Proceedings of the $2^{\text {nd }}$ International Symposium on Wireless Pervasive Computing (ISWPC'07), February, 2007

[21] Elson, J., Girod, L. and Estrin, D. 2002. Fine-grained network time synchronisation using reference broadcasts. In Proceedings of the $5^{\text {th }}$ Symposium on OSDI, December 2002

[22] Ganeriwal, S., Kumar, R. and Srivastava, M. B. 2003. Timing-sync protocol for sensor networks. In Proceedings of ACM SenSys'03, November 2003

[23] Kubisch, M., Karl, H., Wolisz, A., Zhong, L. C. and Rabaey, L. 2003. Distributed algorithms for transmission power control in wireless sensor networks. IEEE WCNS, 2003

[24] Bakshi, A. and Prasanna, V. K. 2004. Structured communication in single hop sensor networks. In Lecture Notes in Computer Science. SpringerLink, 2004

[25] Martinez, M., Padhy, P., Riddoch, A., Ong, H. L. R. and Hart, J. K. 2005. Glacial environment monitoring using sensor networks. REALWSN'05, Stockholm, Sweden, June 2005

[26] Singh, M. and Prasanna, V. K. 2003. Energy-optimal and energybalanced sorting in a single-hop wireless sensor network. In Proceedings of the $1^{\text {st }}$ IEEE PERCOM, 2003

[27] Panta, R. K., Bagchi, S., Khalil, I. and Montestruque, L. 2008. Single versus multi-hop wireless reprogramming in sensor networks. In Proceedings of the $4^{\text {th }}$ International Conference on Testbeds and Research Infrastructures for the Development of Networks \& Communications, 2008

[28] Shnayder, V., Hempstead, M., Chen Bor-rong, Allen, G. W. and Welsh, M. 2004. Simulating the power consumption of large-scale sensor network applications. In Proceedings of ACM SenSys'04, Maryland, USA, November 2004

[29] Srinivasan, K., Dutta, P.,Tavakoli, A. and Levis, P. 2006. Understanding the causes of packet delivery success and failure in dense wireless sensor networks. Technical Report SING-06-00 Stanford University, 2006

[30] Khemapech, I., Duncan, I and Miller, A. 2010. Energy Preservation in Environmental Monitoring WSN. In Proceedings of the IEEE SUTC, California, USA, June 2010

[31] Datasheet - CMR200T and CMR250T. DOI= http://www. citizencrystal.com/images/pdf/k-cmr.pdf 